\title{
Erratum
}

\section{The inverse problem for the Hill operator, a direct approach}

\author{
Pavel Kargaev $^{1}$, Evgeni Korotyaev ${ }^{2}$ \\ 1 Department of Analysis, University of St. Petersburg, Peterhof, 198904, Russia \\ 2 Department of Mathematics 2, 5 Professor Popov Street, Electrotechnical University, \\ St. Petersburg, 197376, Russia
}

Invent. math. 129, 567-593 (1997)

Oblatum 2-IV-1999 \& 27-IV-1999 / Published online: 5 August 1999

The proofs of the main theorems were based on Theorem 5.1 about nonlinear functional analysis. In this abstract theorem we claim that the mapping $f: H \rightarrow H_{1}$ is an isomorphism. Unfortunately, this theorem is not correct and we have to add the following assumption

Condition v). There exists a linear isomorphism $J: H \rightarrow H_{1}$ such that $\left(f(V)-J V, e_{n}\right)_{1}=O(1 / n)$, as $n \rightarrow \infty$ uniformly on bounded subsets of $H$.

Additional part in the proof of Theorem 5.1. We must show that the topology on $M_{m}$, generated by the norm $\|\cdot\|$, coincides with the topology on $M_{m}$ induced by the weak topology of $H$, that is, weak convergence implies strong convergence.

First, we prove the compactness of the mapping $F \equiv f-J$. Indeed, each component $F_{n}(\cdot) \equiv\left(F(\cdot), e_{n}\right)$ is compact since $\left(f(\cdot), e_{n}\right)$ and $\left(J \cdot, e_{n}\right)$ are compact. Then using Condition v) we deduce that $F: H \rightarrow H_{1}$ is compact. Second, assume that $V_{p} \rightarrow V_{0}$ weakly, as $p \rightarrow \infty$ and $V_{p} \in M_{m}, f\left(V_{p}\right) \in$ $K_{m}, p \geq 1$. Then by Condition iv), $y_{p} \equiv f\left(V_{p}\right) \rightarrow y_{0}=f\left(V_{0}\right)$ strongly. We have $y_{p}=J V_{p}+F\left(V_{p}\right)$ and hence $V_{p}=J^{-1}\left(y_{p}-F\left(V_{p}\right)\right) \rightarrow V_{0}$ strongly.

The main results remain true since, in fact, we checked Condition v) in our paper. Indeed, relations (3.38), (3.20), (3.30), (3.25), (3.10-11) show that the mappings $h, l, \mu, M, L$ satisfy Condition v).

The authors would like to thank Prof. J. Voigt for pointing out the error. 